\title{
Repetitive and Restricted Behaviours and Anxiety in Autism Spectrum Disorder: Protocol for a Systematic Review and Meta-Analysis
}

Tegan Sellick ( $\nabla$ teganjsellick@gmail.com )

Monash University https://orcid.org/0000-0003-0442-0048

Alexandra Ure

Monash University

Katrina Williams

Monash University

\section{Protocol}

Keywords: Autism Spectrum Disorder, repetitive and restricted behaviours, anxiety, insistence on sameness, hyperreactivity to sensory input, hyporeactivity to sensory input, restricted interests, repetitive movements, systematic review, meta-analysis

Posted Date: October 7th, 2020

DOl: https://doi.org/10.21203/rs.3.rs-84853/v1

License: (c) (i) This work is licensed under a Creative Commons Attribution 4.0 International License. Read Full License

Version of Record: A version of this preprint was published at Systematic Reviews on December 1st, 2021. See the published version at https://doi.org/10.1186/s13643-021-01830-2. 


\section{Abstract}

\section{Background}

Autism Spectrum Disorder (ASD) is a neurodevelopmental disorder defined by persistent deficits in social functioning and the presence of restricted and repetitive behaviours (RRBs). RRBs refer to four subtypes of behaviour including repetitive movements, speech or use of objects; insistence on sameness; restricted interests; and sensory processing abnormalities. Many individuals with ASD also experience anxiety, which compounds ASD related difficulties and inhibits daily functioning. RRBs have been found to be positively associated with anxiety, however our understanding of the interplay between RRB subtypes and anxiety remains unclear. Thus, the current review aims to systematically review and meta-analyse the association between RRBs and anxiety.

\section{Methods}

This review will collate studies which analyse the association between RRBs and anxiety in individuals with ASD. We will search five databases: CINAHL Plus, Cochrane Central Register of Controlled Trials, Ovid MEDLINE, PsycINFO, and Scopus. Articles included in the review will have their titles, abstracts and full texts reviewed by two independent authors and their risk of bias assessed via the modified Newcastle-Ottawa Scale.

\section{Discussion}

This will be the first review to examine the association between anxiety and the four subtypes of RRBs in individuals with ASD. Understanding this relationship, and the factors associated with this, may help clinicians understand the different underpinnings and presentations of anxiety within this population with potential implications for assessment and treatment.

\section{Systematic Review Registration}

PROSPERO CRD42020185434

\section{Background}

Autism Spectrum Disorder (ASD) is a neurodevelopmental disorder defined by persistent deficits in social functioning and the presence of restricted and repetitive behaviours (RRBs)(1). RRBs refer to four subtypes of behaviour including repetitive movements, speech or use of objects; insistence on sameness; restricted interests; and sensory processing abnormalities (1). Additionally, many individuals with ASD experience elevated levels of anxiety compared to the general population with approximately $40 \%$ of children with ASD meeting the criteria for an anxiety disorder (2) \{van Steensel, 2011 \#36\}and others displaying subclinical symptoms (3). The presence of anxiety negatively impacts one's quality of life by inhibiting daily functioning and increasing the risk of comorbid medical and psychiatric conditions (4). These deficits are further compounded in individuals with ASD as anxiety can be triggered by or 
exacerbate the social difficulties inherent in the disorder resulting in significant distress, problematic behaviours, and compounding relationship difficulties with family members, teachers, and peers $(5,6)$.

Despite these adverse effects, diagnosing and treating anxiety in individuals with ASD is challenging. This is due, in part, to the overlap that exists between anxiety symptoms and autistic traits with the core features of ASD - social communication deficits and RRBs - closely resembling the symptoms of social anxiety and Obsessive Compulsive Disorder (OCD) respectively (7). The diagnosis and treatment of anxiety is further complicated by its atypical presentation in individuals with ASD. Anticipatory worry related to changes in routine; sensory issues; unusual fears; and social aversion that is not linked to fears of rejection are often experienced by individuals with ASD alongside traditional anxiety symptoms (8). This atypical presentation, paired with overlapping symptomology, may result in anxiety being incorrectly diagnosed or remaining undetected and subsequently untreated (9). Either is potentially detrimental, as anxiety symptoms in youth significantly predict later anxiety disorders (10) and a diagnosis of anxiety directs intervention.

\section{RRBs and Anxiety}

There is a body of evidence highlighting the association between RRBs and anxiety symptoms in individuals with ASD (11). Specifically, cross-sectional studies have found moderate to large correlations between the two constructs $(r=.41-.69)$ with individuals experiencing higher anxiety engaging in more RRBs (12-14). Several longitudinal studies exploring the nature of this relationship have found that the severity of RRBs predicts later anxiety, suggesting that a unidirectional relationship exists between the two constructs $(p<.001-p=0.026)(11,15,16)$. The strong and consistent associations found across studies suggests the RRBs and anxiety are interrelated; that this relationship is specific to children with ASD (14); and that the presence of RRB's proceeds the onset of anxiety $(11,15,16)$. Subsequently, RRBs have been conceptualised as an early manifestation of anxiety $(11,15)$ or a maladaptive response to negative affect which subsequently increases one's risk of anxiety over time $(10,11,17)$. Although engaging in RRBs may temporarily reduce anxiety symptoms, their continued use may impede one's ability to adopt more adaptative coping mechanisms, reduce their ability to engage in their environment, and therefore ultimately perpetuate feelings of anxiety $(10,11,17)$. This parallels the trajectory observed in OCD whereby individuals engage in compulsions to neutralise anxiety associated with obsessions (4, 17). However, in reality, these compulsions add considerable distress to individuals with OCD as they do not address the source of anxiety and interfere with daily functioning $(4,17)$. As the hypothesised relationship between anxiety and RRBs in ASD is thought to mirror the relationship between obsessions and compulsions in the OCD literature, it is plausible to suggest that RRBs could be an observable indicator of future anxiety. This could help clinicians identify and treat anxiety in young children, preventing the manifestation of significant distress in later years.

\section{RRB Subtypes and Anxiety}

Although the literature consistently reports that RRBs are associated with anxiety, it is important to clarify if this relationship exists for all or specific RRB subtypes. There is a consistent body of evidence to 
suggest that insistence on sameness and restricted interests are linked to anxiety (3). However, the relationship between the remaining subtypes and anxiety remains unclear, with conflicting evidence emerging from the literature. This lack of clarity may be associated with the varying definitions of RRBs. Whilst some studies have explored subtypes individually $(3,18)$, others have combined the subtypes into two broad domains known as low-order and high-order RRBs $(10,19)$. Low-order RRBs refer to repetitive movements that may be motivated by sensory feedback, whereas high-order RRBs include restricted interests and insistence on sameness behaviours (20). These domains have been criticised for being too broad and therefore disregarding important differences in the functions served by RRB subtypes (3). The grouping of low-order RRBs is problematic as they are thought to have contradictory functions. For instance, sensory processing abnormalities may increase one's risk of experiencing anxiety as having a low threshold for sensory input is thought to exacerbate environmental stressors (16). This is consistent with the finding that hyperactivity to sensory input in toddlers predicts later anxiety (16).

Contrastingly, repetitive movements are thought to enable one to manage anxiety that stems from sensory processing abnormalities (21). A discrepancy is therefore highlighted between the two low-order RRBs, with one thought to contribute to anxiety, whilst the other a response.

Subtypes of high-order RRBs are also understood to uniquely moderate anxiety symptoms. For instance, restricted interests are thought to be a maladaptive coping response to anxiety (17), whilst insistence on sameness behaviours may enable one to control their environment and thus buffer the effect of anxiety (21). These distinctions are consistent with results of factor analyses which have found restricted interests and insistence on sameness behaviours to be distinct subtypes (22-24). Thus, it is critical to examine these subtypes individually in order to clarify their differential relationship with anxiety. This knowledge could help clinicians tailor diagnostic tools and treatment interventions as specific RRBs may be utilised as a marker for anxiety, whilst others could be targeted for interventions.

\section{The Relationship between RRB Subtypes}

To effectively conceptualise the associations between RRBs and anxiety, it is important to also understand the inter-relationships amongst RRB subtypes. There is emerging evidence to suggest that RRBs subtypes are inter-related $(21,25)$. Specifically, sensory processing abnormalities have been shown to negatively correlate with repetitive movements and high-order RRBs, whilst a positive association has been observed between repetitive movements and high-order RRBs $(21,25)$. However, not all studies yield consistent results with Lidstone (12) finding that low registration (a measure of hyporeactivity) and sensory sensitivity (a measure of hyperreactivity) were not associated with repetitive movements.

\section{Factors influencing the RRB-Anxiety relationship}

The relationship between specific RRB subtypes and anxiety may be influenced by the presence of additional RRBs. Wigham (21) reported that anxiety, in combination with intolerance of uncertainty, mediated the relationship between sensory processing and high-order RRBs. Further study has reported a path from hyporeactivity, but not hyper-reactivity, to high-order RRBs through anxiety alone (25). These 
findings support the hypothesis that restricted interests and insistence on sameness behaviours enable individuals to reduce or avoid anxiety associated with sensory input $(12,21)$. However, in contrast, anxiety did not mediate the relationship between sensory processing abnormalities and repetitive movements $(21,25)$. Given that different studies provide conflicting evidence about the relationship between RRB subtypes, it is critical to explore this in the context of the anxiety-RRB relationship. Collating the associations between RRB subtypes may help clinicians understand if these behaviours are interrelated and whether clusters or specific RRBs are associated with anxiety.

Other individual traits or characteristics may also influence the relationship between RRBs and anxiety. For instance, intolerance of uncertainty, a trait associated with high levels of worry and avoidance when faced with uncertainty, has been linked to both anxiety and RRBs $(20,21,26)$. Hwang (26) found that intolerance of uncertainty mediated the relationship between anxiety and all RRB subtypes except for repetitive movements. Age and intelligence quotient (IQ) have also been identified as factors relating to both anxiety and RRB subtypes $(3,27)$, however the role they play within the RRB-anxiety relationship remains unclear. Findings to date are mixed with some studies finding that anxiety increases with age but not with RRBs $(16,28)$, whilst others have found the reverse (26). As all individuals with ASD present with at least two RRBs as per the Diagnostic and Statistical Manual of Mental Disorders (DSM: 1), identifying and collating the factors associated with the RRB-anxiety relationships could help clinicians identify who are most vulnerable to developing anxiety symptoms.

\section{Current Study}

Our systematic review aims to: 1) meta-analytically assess the association between RRB subtypes and anxiety symptoms in individuals with ASD; 2 ) meta-analytically assess the association between the RRB subtypes in the context of the anxiety-RRB relationship; and 3) collate factors associated with both RRBs and anxiety symptoms. These aims will be addressed via the following research questions:

1. What is the association between RRB subtypes and anxiety symptoms in people with ASD?

2. In studies exploring RRBs and anxiety in people with ASD, what is the association between the RRBs subtypes?

3. For individuals with ASD, what are the factors associated with both RRBs and anxiety?

This review is registered on the PROSPERO database (CRD42020185434).

\section{Methods}

\section{Eligibility Criteria}

Observational studies will be included in the review if they meet the following eligibility criteria:

1. The sample consists of participants with a diagnosis of ASD under the DSM (DSM-III, DSM-III-R, DSM-IV, DSM-IV-TR, or DSM-5), International Classification of Diseases (ICD: ICD-9, ICD-10, or ICD-11) 
or an equivalent diagnosis from the Autism Diagnostic Interview - Revised, Autism Diagnostic Observation Schedule, or Childhood Autism Rating Scale.

2. Studies include at least one standardised measure of anxiety completed by the participant or their parent. This includes measures of anxiety symptoms and disorders as defined by the DSM-5, as well as OCD.

3. Studies include at least one standardised measure of RRBs completed by the participant or their parent.

4. The association between anxiety and RRBs, or an RRB subtype, was analysed or relevant data is reported and available for analysis.

5. Was published in or after 1987 when RRBs first entered the DSM-III-R criteria for 'Autistic Disorder'.

6. Studies are written in English.

Articles will be excluded if they include less than five participants or if they are an opinion piece, letter, editorial or review. The reference list of relevant reviews will be checked.

\section{Search Strategy}

The literature search will be conducted across five databases: CINAHL Plus, Cochrane Central Register of Controlled Trials, Ovid MEDLINE, PsycINFO, and Scopus. The search strategy was developed alongside a senior tertiary librarian to identify studies which meet the above eligibility criteria. The reference list of all eligible articles will also be searched to ensure literature saturation. The search strategy for Ovid MEDLINE is included as an additional file (Additional file 1).

Unpublished materials will also be identified by searching clinical trial registries, ClinicalTrials.gov and Australian New Zealand Clinical Trials Registry (ANZCTR), and theses databases, Trove and ProQuest Dissertations and Theses Global. An advanced Google search will also be completed to identify papers that were not included in the databases.

The search will be limited to humans.

\section{Study Selection}

Articles identified from the literature search will be exported into Endnote X9 and duplicates will be removed.

The titles and abstracts of remaining articles will be imported into Covidence and their eligibility will be assessed by two independent reviewers. Eligible articles will have this process repeated for the full article. If an article is missing information, the author will be asked to provide this information.

Reviewers will solve disagreements by discussing the matter in relation to the inclusion criteria. If consensus is not reached, a third reviewer will be engaged to resolve this. 
A PRISMA flow diagram will highlight the studies remaining at each stage.

\section{Data Extraction}

A standardised form will be utilised by one reviewer to extract the following data for each study: (1) study details; (2) sample size; (3) participant demographics; (4) how ASD diagnoses were confirmed; (5) anxiety type/s investigated; (6) anxiety measure/s used; (7) RRB subtype/s investigated; (8) RRB measure/s used; (9) factors associated with RRBs and anxiety; (10) reported outcomes, statistical analyses, adjustments, and significance; and (11) information for assessing risk of bias.

If overlapping studies are identified, we will include the study with the largest sample size to avoid including duplicate samples or studies as this may bias the results.

When data is missing, we will contact the first author of the study via email and ask them to provide this information. If this information is not provided, we will aim to retain the study in the review. However, a sensitivity analysis will be run to assess the impact of missing data on the results.

\section{Risk of Bias Assessment}

We will use a modified version Newcastle-Ottawa Scale to conduct a risk of bias assessment for each included study (29). One reviewer will conduct the risk of bias assessment to assess selection, comparability, and outcome across all studies.

\section{Data Analysis and Synthesis}

\section{Research Questions 1 \& 2}

For each study, the association between anxiety symptoms and RRB subtypes and the association between different RRB subtypes will be reported, including whether the association is unadjusted or adjusted. If the association is adjusted, we will also report the covariates included in the analyses. Categorical (Odds Ratio (OR) or Relative Risks (RR)) and continuous (Pearson's correlation coefficient (r), Cohen's d or outcomes of linear regression) outcomes are expected. If there are two or more studies assessing the association of one RRB and anxiety or between one RRB and another RRB, data will be standardised to allow for a more direct comparison (30).

Homogeneity will be assessed by inspecting the forest plots and $\mathrm{I}^{2}$ statistic. If the studies are homogenous, a random effects meta-analysis will be conducted to assess the magnitude of the association between anxiety and each RRB subtype. Additionally, subgroup analyses will be conducted to investigate important differences based on: (1) age; (2) level of functioning; (3) diagnostic characteristics of ASD; (4) IQ or Developmental Quotient; (5) mental health diagnoses other than anxiety; and (6) anxiety diagnoses. 
A sensitivity analysis will also be run to assess the potential impact of: (1) methodological differences across studies; (2) risk of bias across studies; (3) missing data across studies; and (4) outliers.

Publication bias will be assessed via an examination of funnel plots and Egger's test.

If there are fewer than two studies for each association of interest, or if studies are not homogenous, we will present a narrative synthesis.

\section{Research Question 3: For individuals with ASD, what are the factors associated with both RRBs and anxiety?}

We will collate and describe the factors reported to be associated with RRBs and anxiety. Each factor, whether it is significantly associated with the construct, and the associations will be recorded. Whether a factor significantly mediates or moderates the anxiety-RRB relationship will also be reported.

This methodology was developed using the Preferred Reporting Items for Systematic Review and MetaAnalysis Protocols (PRISMA-P) (31).

\section{Discussion}

This review aims to clarify the associations between RRBs and anxiety in individuals with ASD. Improving our understanding of this relationship, and the related interpersonal characteristics influencing this relationship, may help clinicians understand the different underpinnings and presentations of anxiety within this population. This will assist accurate diagnosis and tailoring interventions to improve treatment outcomes. It is possible that specific subtypes of RRBs may be identified and used as an observable marker of anxiety or a point for intervention. Given the current challenges in assessing anxiety in this population, this could help alleviate its long term psychological and social effects.

\section{Amendments to the protocol}

The protocol published with PROSPERO had undergone one amendment prior to the submission of this manuscript for publication. The amendments include:

- Searching a second clinical trial database to improve the comprehensiveness of the search;

- Expanding the inclusion criteria to include Obsessive Compulsive Disorder as an anxiety disorder due to its historical link with anxiety;

- Stating that we will not re-run our search prior to the final analysis as the analysis will take place within one year of the initial search;

- No longer contacting prominent authors in the field searching for articles as we have already executed a comprehensive search strategy;

- Having one author extract data and conduct the risk of bias assessment; 
- Renaming the field 'study design' to 'study details' in our data extraction form to capture more information. Additionally, the fields 'RRB definition' and 'RRB subtype/s investigated' have been merged;

- Including standardised beta from linear regressions as an outcome measure;

- Conducting a sensitivity analysis on any missing data.

Any future amendments to the protocol will also be registered with PROSPERO and available online.

\section{Abbreviations}

ANZCTR: Australian New Zealand Clinical Trials Registry

APA: American Psychiatric Association

ASD: Autism Spectrum Disorder

DSM: Diagnostic and Statistical Manual of Mental Disorders

ICD: International Classification of Diseases

IQ: Intelligence Quotient

OCD: Obsessive Compulsive Disorder

OR: Odds Ratio

PRISMA-P: Preferred Reporting Items for Systematic Review and Meta-Analysis Protocols

RR: Relative Risks

RRBs: Restricted and Repetitive Behaviours

\section{Declarations}

Ethics approval and consent to participate

Not applicable.

\section{Consent for publication}

Not applicable.

\section{Availability of data and materials}

The search strategy for Ovid MEDLINE can be found as an Additional File. 


\section{Competing interests}

The authors declare that they have no competing interests.

\section{Funding}

There is no funding being provided for this systematic review. The first author is undertaking the review as part of her honours degree through Monash University, Australia. Professor Katrina Williams and Dr. Alexandra Ure, as honours supervisors, will review and contribute as required.

\section{Authors' contributions}

TS wrote the first draft of the protocol. AU and KW contributed to the editing process prior to submission to the Journal of Systematic Reviews. TS and AU will screen all abstracts and titles and full-text articles following collation of database search results. TS will also assess the bias of articles prior to data extraction. TS will be responsible for the data extraction. All authors have read and approved the final manuscript.

\section{Acknowledgements}

We acknowledge Anne Young, Monash University librarian, for providing assistance in the development of the search strategies.

\section{References}

1. American Psychiatric Association. Diagnostic and statistical manual of mental disorders: DSM-5. 5th ed. Arlington, VA: American Psychiatric Association; 2013.

2. van Steensel FJA, Bögels SM, Perrin S. Anxiety disorders in children and adolescents with autistic spectrum disorders: A meta-analysis. Clin Child Fam Psychol Rev. 2011;14(3):302-17.

3. Russell K, Frost K, Ingersoll B. The relationship between subtypes of repetitive behaviors and anxiety in children with autism spectrum disorder. Res Autism Spectr Disord. 2019;62:48-54.

4. Kring AM, Kyrios M, Fassnacht D, Lambros A, Mihaljcic T, Teesson M. Abnormal psychology. Milton, QLD: Wiley; 2018.

5. Vasa R, Carroll L, Nozzolillo A, Mahajan R, Mazurek M, Bennett A, et al. A systematic review of treatments for anxiety in youth with autism spectrum disorders. J Autism Dev Disord. 2014;44(12):3215-29.

6. Top DN, Jr., Luke SG, Stephenson KG, South M. Psychophysiological arousal and auditory sensitivity in a cross-clinical sample of autistic and non-autistic anxious adults. Front Psychiatry. 2019;9:1-12.

7. Zaboski BA, Storch EA. Comorbid autism spectrum disorder and anxiety disorders: A brief review. Future neurol. 2018;13(1):31-7. 
8. Kerns CM, Kendall PC, Berry L, Souders MC, Franklin ME, Schultz RT, et al. Traditional and atypical presentations of anxiety in youth with autism spectrum disorder. J Autism Dev Disord. 2014:44(11):2851-61.

9. Adams D, Young K, Simpson K, Keen D. Parent descriptions of the presentation and management of anxiousness in children on the autism spectrum. Autism. 2019;23(4):980-92.

10. Rodgers J, Glod M, Connolly B, McConachie $H$. The relationship between anxiety and repetitive behaviours in autism spectrum disorder. J Autism Dev Disord. 2012;42(11):2404-9.

11. Baribeau DA, Vigod S, Pullenayegum E, Kerns CM, Mirenda P, Smith IM, et al. Repetitive behavior severity as an early indicator of risk for elevated anxiety symptoms in autism spectrum disorder. $J$ Am Acad Child Adolesc Psychiatry. 2020;59(7):890-9.

12. Lidstone J, Uljarević M, Sullivan J, Rodgers J, McConachie H, Freeston M, et al. Relations among restricted and repetitive behaviors, anxiety and sensory features in children with autism spectrum disorders. Res Autism Spectr Disord. 2014;8(2):82-92.

13. Muskett A, Capriola-Hall N, Radtke S, Factor R, Scarpa A. Repetitive behaviors in autism spectrum disorder: Associations with depression and anxiety symptoms. Res Autism Spectr Disord. 2019;68.

14. Rodgers J, Riby DM, Janes E, Connolly B, McConachie H. Anxiety and repetitive behaviours in autism spectrum disorders and williams syndrome: A cross-syndrome comparison. J Autism Dev Disord. 2012;42(2):175-80.

15. Baird K, Szatmari, P., Georgiades, S., Duku, E., Bryson, S., Fom- bonne, E., Smith, I., Vaillancourt, T., Volden, J., Waddell C., Zwaigenbaum, L. Investigating the association between anxiety and fixed interests, repetitive behaviours in preschool children with ASD. Paper presented at: International Meeting for Autism Research; 2012; Ontario, Canada.

16. Green SA, Ben-Sasson A, Soto TW, Carter AS. Anxiety and sensory over-responsivity in toddlers with autism spectrum disorders: bidirectional effects across time. J Autism Dev Disord. 2012;42(6):11129.

17. Spiker MA, Lin CE, Van Dyke M, Wood JJ. Restricted interests and anxiety in children with autism. Autism. 2012;16(3):306-20.

18. Mazurek M, Vasa R, Kalb L, Kanne S, Rosenberg D, Keefer A, et al. Anxiety, sensory over-responsivity, and gastrointestinal problems in children with autism spectrum disorders. J Autism Dev Disord. 2013;41(1):165-76.

19. Factor R, Condy E, Farley J, Scarpa A. Brief Report: Insistence on sameness, anxiety, and social motivation in children with autism spectrum disorder. J Autism Dev Disord. 2016;46(7):2548-54.

20. Joyce C, Honey E, Leekam SR, Barrett SL, Rodgers J. Anxiety, intolerance of uncertainty and restricted and repetitive behaviour: Insights directly from young people with ASD. J Autism Dev Disord. 2017;47(12):3789-802.

21. Wigham S, Rodgers J, South M, McConachie H, Freeston M. The interplay between sensory processing abnormalities, intolerance of uncertainty, anxiety and restricted and repetitive behaviours in autism spectrum disorder. J Autism Dev Disord. 2015;45(4):943-52. 
22. Bishop S, Hus V, Duncan A, Huerta M, Gotham K, Pickles A, et al. Subcategories of restricted and repetitive behaviors in children with autism spectrum disorders. J Autism Dev Disord. 2013;43(6):1287-97.

23. Hooker JL, Dow D, Morgan L, Schatschneider C, Wetherby AM. Psychometric analysis of the repetitive behavior scale-revised using confirmatory factor analysis in children with autism. Autism Res. 2019;12(9):1399-410.

24. Lam KSL, Bodfish JW, Piven J. Evidence for three subtypes of repetitive behavior in autism that differ in familiality and association with other symptoms. J Child Psychol Psychiatry. 2008;49(11):1193200.

25. Glod M, Riby DM, Rodgers J. Short report: Relationships between sensory processing, repetitive behaviors, anxiety, and intolerance of uncertainty in autism spectrum disorder and Williams syndrome. Autism Res. 2019;12(5):759-65.

26. Hwang YI, Arnold S, Srasuebkul P, Trollor J. Understanding anxiety in adults on the autism spectrum: An investigation of its relationship with intolerance of uncertainty, sensory sensitivities and repetitive behaviours. Autism. 2020;24(2):411-22.

27. Gotham K, Bishop SL, Hus V, Huerta M, Lund S, Buja A, et al. Exploring the relationship between anxiety and insistence on sameness in autism spectrum disorders. Autism Res. 2013;6(1):33-41.

28. Maclennan K, Roach L, Tavassoli $T$. The relationship between sensory reactivity differences and anxiety subtypes in autistic children. Autism Res. 2020;13(5):785-95.

29. Modesti PA, Reboldi G, Cappuccio FP, Agyemang C, Remuzzi G, Rapi S, et al. Panethnic differences in blood pressure in europe: A systematic review and meta-analysis. PLoS ONE. 2016;11(1):e0147601.

30. Higgins JPT, Thomas J, Chandler J, Cumpston M, Li T, Page MJ, et al. Cochrane handbook for systematic reviews of interventions. 2nd ed. Hoboken, NJ: Wiley-Blackwell; 2019.

31. Shamseer L, Moher D, Clarke M, Ghersi D, Liberati A, Petticrew M, et al. Preferred reporting items for systematic review and meta-analysis protocols (PRISMA-P) 2015: Elaboration and explanation. British Medical Journal. 2015;349(jan02 1).

\section{Supplementary Files}

This is a list of supplementary files associated with this preprint. Click to download.

- PRISMAPchecklist.docx

- OVIDMedline.docx 